\title{
Activation of the ERK/Creb/Bcl-2 pathway protects periodontal ligament stem cells against hydrogen peroxide-induced oxidative stress
}

\author{
XIAOHUI FU ${ }^{1}$, YIMIAO FENG ${ }^{2}$, BINGYI SHAO ${ }^{3}$ and YANZHEN ZHANG ${ }^{1}$
}

\begin{abstract}
Departments of ${ }^{1}$ General Dentistry and ${ }^{2}$ Orthodontics, The Second Affiliated Hospital, College of Medicine, Zhejiang University, Hangzhou, Zhejiang 310009; ${ }^{3}$ Department of Operative Dentistry and Endodontics, The Affiliated Hospital of Stomatology, Chongqing Medical University, Chongqing 400015, P.R. China
\end{abstract}

Received May 2, 2018; Accepted February 22, 2019

DOI: $10.3892 / \mathrm{mmr} .2019 .10027$

\begin{abstract}
Periodontal ligament stem cells (PDLSCs) are promising stem cells sources for regenerative medicine, particularly clinical periodontal ligament repair. It is critical to maintain high quality and a large quantity of PDLSCs for clinical usage. However, how PDLSCs respond to environmental stimuli, including reactive oxygen species (ROS), is poorly understood. The aim of the present study was to investigate how PDLSCs react to oxidative stress and the underlying mechanisms. Hydrogen peroxide-induced oxidative stress was used to mimic a ROS increase in rat PDLSCs. The expression levels of Creb were detected under oxidative stress to examine the role that Creb serves in PDLSCs under oxidative stress. The present results demonstrated that the expression of Creb was reduced in a dose-dependent manner in response to the $\mathrm{H}_{2} \mathrm{O}_{2}$ stimulus. Overexpressing Creb significantly reduced the ROS levels and protein expression levels of apoptotic genes in PDLSCs. The phosphorylation of the ERK pathway is indispensable in the activation of Creb-induced protection. Our results revealed a protective role of Creb in ROS-induced apoptosis, and validated the ERK/Creb/apoptosis regulator Bcl-2 pathway works as an anti-apoptotic signaling in PDLSCs. These findings will facilitate the in vitro culturing of PDLSCs for clinical usage and promote stem cell based therapy for periodontal tissue regeneration.
\end{abstract}

Correspondence to: Professor Yanzhen Zhang, Department of General Dentistry, The Second Affiliated Hospital, College of Medicine, Zhejiang University, 88 Jiefang Road, Hangzhou, Zhejiang 310009, P.R. China

E-mail: 2191004@zju.edu.cn

Abbreviations: Creb, cAMP responsive element binding protein; PDLSCs, periodontal ligament stem cells; ROS, reactive oxygen species; ERK, extracellular signal regulated kinase; DCFDA, 2',7'-dichlorodihydrofluorescein diacetate

Key words: Creb, PDLSCs, ERK, ROS, apoptosis

\section{Introduction}

Periodontal diseases are one of the most common diseases worldwide and include the inflammatory disorders gingivitis and periodontitis (1). Besides conventional treatment that controls inflammation, stem cell-based regeneration of periodontal ligaments and other periodontal tissues brings new hope for patients suffering from severe periodontal diseases $(2,3)$. Among the various types of adult multipotent cells, periodontal ligament stem cells (PDLSCs) are multipotent stem cells that are capable of differentiating into alveolar bone, cementum, peripheral nerves and even blood vessels (4-8). However, the amounts of residual PDLSCs are limited and in vitro expanded PDLSCs may serve as an alternative source of stem cells for applications in the regeneration of periodontal tissues. External stimuli and genetic backgrounds, including hypoxia, hormones, histone modification and non-coding RNAs, affect the quality and function of PDLSCs (9-15). Oxidative stress is characterized by misregulated reactive oxygen species (ROS) which are primarily generated from mitochondrial complexes I and III. A basal level of ROS is required for normal cell activities. However, if free radicals are abnormally accumulated, important biomolecules including DNA and proteins are negatively affected and the survival of stem cells is compromised $(16,17)$. Although oxidative stress is critical for stem cell aging and survival (18-20), it is not clear whether oxidative stress impacts the molecular regulation of PDLSCs in vitro.

cAMP responsive element binding protein (Creb) was initially identified within the rat brain as a transcription factor, and the phosphorylation of Creb at serine 133 is required for its transcriptional activity (21-24). Since this discovery, various functions of Creb have been described and $>4,000$ genes contain the cAMP responsive element in their promoter regions (25). Creb regulates cell proliferation, differentiation and survival, in addition to inflammation, in various type of cells (26). Since Creb is stimulated by various extracellular signals, such as hypoxia and hormones, it is of interest to examine the role of Creb in PDLSCs (25).

Mitogen-activated protein kinase/extracellular signal-regulated kinase (MAPK/ERK) phosphorylates 
Creb at SER 133 via ribosomal S6 kinase $(27,28)$. Previous reports suggest that the ERK1/2 pathway is required for PDLSC differentiation $(29,30)$ suggesting that ERK-mediated phosphorylation of Creb may regulate PDLSCs. Of note, administration of $\mathrm{H}_{2} \mathrm{O}_{2}$ increases the phosphorylation level of ERK1/2, but reduces the phosphorylation level of Creb at SER 133, suggesting that ERK-Creb signaling is modulated by oxidative stress in PC12 cells (31). A recent report demonstrated that Creb/ERK signaling inhibits oxidative stress in osteoblast cells (32). Evidence also suggests that ERK-dependent Creb activation reduces apoptosis in human neuroblastoma cells (33). Thus, it is possible that the ERK/Creb pathway regulates PDLSCs in an oxidative stress-dependent manner and controls the apoptosis of PDLSCs.

Phosphorylated Creb is able to bind directly to the apoptosis regulator $\mathrm{Bcl}-2$ (Bcl-2) promoter and induce the transcription of Bcl-2, which in turn rescues B cells from apoptosis (34). In addition, Creb-mediated $\mathrm{Bcl}-2$ expression also protects Hela cells from apoptosis (35). Bcl-2 is an anti-apoptotic regulator which negatively controls the apoptosis process by inhibiting cytochrome $c$ release, while apoptosis regulator BAX is a pro-apoptotic regulator which facilitates the release of cytochrome $c$ (36). Selenite inhibits the phosphorylation of Creb and reduces the level of Bcl-2, resulting in apoptosis, whereas apricot increases the expression of Bcl-2 and Creb and attenuates oxidative stress $(37,38)$. U0126 is a specific inhibitor of dual specificity mitogen-activated protein kinase kinase 1 , an upstream kinase of ERK1/2 $(39,40)$. By using U0126, it may be possible to validate the association between ERK and Creb. These previous results indicated that Creb-mediated $\mathrm{Bcl}-2$ expression is critical for oxidative stress and apoptosis, suggesting that the $\mathrm{Creb} / \mathrm{Bcl}-2$ pathway may be involved in regulating PDLSCs.

The present study aimed to reveal the role of Creb in $\mathrm{H}_{2} \mathrm{O}_{2}$-induced oxidative stress in PDLSCs. The expression alteration of Creb upon administration of $\mathrm{H}_{2} \mathrm{O}_{2}$ was assessed. $\mathrm{Creb}$ was overexpressed in PDLSCs to study its function. The ROS level in wild-type PDLSCs and PDLSCs overexpressing Creb was also monitored under treatment with $\mathrm{H}_{2} \mathrm{O}_{2}$. To reveal the mechanism of Creb-mediated PDLSC protection, ERK/Creb/Bcl-2 signaling was examined. The results revealed a protective role of Creb against $\mathrm{H}_{2} \mathrm{O}_{2}$-induced oxidative stress, and provided evidences for the further application of PDLSCs in regenerative medicine.

\section{Materials and methods}

Periodontal ligament stem cell isolation and culture. The rat PDLSCs were isolated by modifying a method described previously (41). Five female Wistar rats (age, 8 weeks; weight, $250 \pm 50 \mathrm{~g}$; Zhejiang Center of Laboratory Animals, Hangzhou, China) were housed under standardized conditions: Room temperature, $20-24^{\circ} \mathrm{C}$; relative air humidity, 35-70\%; 12-h light/dark cycle. The animals had free access to standard laboratory food (Zhejiang Center of Laboratory Animals) and sterile water. The rat periodontal ligaments were cut into small pieces and digested in $0.3 \%$ collagenase (Sigma-Aldrich; Merck KGaA, Darmstadt, Germany) at $37^{\circ} \mathrm{C}$ for $4 \mathrm{~h}$. The cells obtained were pooled, seeded and cultured in Dulbecco's modified Eagle's medium (HyClone;
GE Healthcare Life Sciences, Logan, UT, USA) containing 10\% fetal bovine serum (Gibco; Thermo Fisher Scientific, Inc., Waltham, MA, USA) in a humidified atmosphere $(95 \%$ air, $5 \% \mathrm{CO}_{2}$ ) at $37^{\circ} \mathrm{C}$. STRO-1 $1^{+}$PDLSCs were purified using immunomagnetic beads (Invitrogen; Thermo Fisher Scientific, Inc.), according to the manufacturer's protocol. Attracted cells were segregated using a magnetic particle separator following three washes with isolation buffer (PBS with $0.1 \%$ bovine serum albumin (Sigma-Aldrich; Merck KGaA) and $2 \mathrm{mM}$ EDTA). Subsequently cells were seeded and cultured in growth medium in a humidified atmosphere $(95 \%$ air, $5 \%$ $\mathrm{CO}_{2}$ ) at $37^{\circ} \mathrm{C}$.

The animal care and protocols were approved by the Institutional Review Boards of Zhejiang University (Hangzhou, China; approval no. ZJUAC160809012).

$\mathrm{H}_{2} \mathrm{O}_{2}$ treatment. The cells were treated with $62.5,125,250$, 500 and $1,000 \mu \mathrm{M} \mathrm{H}_{2} \mathrm{O}_{2}$ for $24 \mathrm{~h}$ to detect the influence of oxidative stress on Creb expression. Cells treated with an equivalent volume of PBS were used as a control. In subsequent experiments, the cells were treated with $125 \mu \mathrm{M} \mathrm{H}_{2} \mathrm{O}_{2}$ to induce oxidative stress.

For the ERK inhibition assay, $10 \mu \mathrm{M}$ ERK-specific inhibitor U0126 (Promega Corporation, Madison, WI, USA) dissolved in dimethyl sulfoxide (DMSO) was added into the culture medium for $1 \mathrm{~h}$ prior to treatment with $\mathrm{H}_{2} \mathrm{O}_{2}$. An equal volume of DMSO was used as the vehicle control.

Reverse transcription-quantitative polymerase chain reaction $(R T-q P C R)$. Total RNA was extracted from PDLSCs using TRIzol ${ }^{\circledR}$ reagent (Invitrogen; Thermo Fisher Scientific, Inc.) according to the manufacturer's protocol. For each sample, 500 ng total RNA was reverse-transcribed using GoScript ${ }^{\mathrm{TM}}$ RT system (Promega Corporation) according to the manufacturer's protocol. The resulting cDNA was diluted 40 times and RT-qPCR was performed with the Ace Q qPCR SYBR Green Master Mix kit (Vazyme, Piscataway, NJ, USA). PCR amplification was conducted as follows: Preheating at $95^{\circ} \mathrm{C}$ for $5 \mathrm{~min}$, followed by 40 cycles of denaturation at $95^{\circ} \mathrm{C}$ for $10 \mathrm{sec}$ and annealing/extension at $60^{\circ} \mathrm{C}$ for $30 \mathrm{sec}$. Data were quantified using the $2^{-\Delta \Delta \mathrm{Cq}}$ method as previously described (42). The following primers were used in the tests: Rat GAPDH forward, 5'-AACGACCCC TTCATTGACC-3' and reverse, 5'-TCCACGACATACTCAGCA CC-3'; rat Creb forward, 5'-CACATAGCCCAGGTATCC-3' and reverse, 5'-TGAACTGTTTGGACTTGTGG-3'.

Western blotting. Total proteins were extracted from PDLSCs using radioimmunoprecipitation assay lysis buffer (Thermo Fisher Scientific, Inc.) and were quantified using the Pierce Bicinchoninic Acid Protein Assay kit (Thermo Fisher Scientific, Inc.) according to the manufacturers' protocols. Extracted proteins from each sample $(25 \mu \mathrm{g})$ were separated with $10 \%$ SDS-PAGE and transferred onto a nitrocellulose membrane (Thermo Fisher Scientific,Inc.). The membrane was blocked with 5\% milk in TBS with Tween-20 (TBST; Bio-Rad Laboratories, Inc., Hercules, CA, USA) for at least $1 \mathrm{~h}$ at room temperature and subsequently incubated with each primary antibody overnight at $4^{\circ} \mathrm{C}$. Membranes were washed with TBST buffer at least five times for $5 \mathrm{~min}$ and incubated with the secondary antibodies for $1 \mathrm{~h}$ at room temperature. The protein bands were visualized 
using an enhanced chemiluminescence system (Amersham; GE Healthcare, Chicago,IL, USA). Equal loading of samples was verified by immunoblotting for GAPDH (1:10,000, cat. no. ab181602; Abcam, Cambridge, UK). The primary antibodies used in the present study were anti-Creb antibody (1:500, cat. no. ab32515), anti-phospho-Creb antibody (1:5,000, cat. no. ab32096), anti-Bcl-2 antibody (1:500, cat. no. ab59348), anti-caspase-9 (1:500, cat. no. ab25758), anti-ERK (1:1,000, cat. no. ab17942) and anti-phospho-ERK (1:1,000, cat. no. ab201015) (all from Abcam). All primary antibodies were further labeled with horseradish peroxidase-conjugated goat anti-rabbit secondary antibody (1:5,000, cat. no. ab6721; Abcam). Densitometric quantification was performed using ImageJ software (version 1.46; National Institutes of Health, Bethesda, MD, USA).

MTT assays. PDLSCs treated with various concentrations of $\mathrm{H}_{2} \mathrm{O}_{2}$ for $24 \mathrm{~h}$ were plated at a density of $1 \times 10^{4}$ cells/well in a 96-well plate. Viable cells were stained with $0.25 \mathrm{mg} / \mathrm{ml}$ MTT for $1 \mathrm{~h}$ at $37^{\circ} \mathrm{C}$. The media were then removed, and formazan crystals produced were dissolved by the addition of DMSO (absorbance, $540 \mathrm{~nm}$ ).

Creb overexpression. A plasmid based on the pcDNA3.1 backbone (Addgene, Inc., Cambridge, MA, USA) overexpressing Creb was obtained by cloning full-length human Creb cDNA by PCR with the following primers: Forward, 5'-GGGAAT TCCCGGTAACTAAATG-3' and reverse, 5'-GGGCGGCCG CTTGTCTTTCAGGTTGTGG-3'. The primers were designed according to the Creb sequence provided by the National Center for Biotechnology Information database (GeneBank ID: AY347527.1; www.ncbi.nlm.nih.gov/genbank). The resultant cDNA was verified by sequencing (Beijing Genomics Institute, Beijing, China) and inserted into the backbone plasmid between the NotI and EcoRI cloning site. Plasmid pcDNA3.1 was used as a vector control. PDLSCs were seeded in 60-mm dishes at $5 \times 10^{5}$ cells/dish and incubated overnight prior to transfection with $1 \mu \mathrm{g}$ plasmid DNA using jetPRIMETM DNA and transfection reagent (VWR International, Ltd., Lutterworth, UK), according to the manufacturer's protocol. After $24 \mathrm{~h}$, the cell lysates were collected for western blotting or RT-qPCR.

Measurement of ROS. The intracellular ROS level was measured via the oxidation of the ROS-sensitive fluorophore 2',7'-dichlorodihydrofluorescein diacetate (DCFDA; Thermo Fisher Scientific, Inc.), according to the manufacturer's protocol. PDLSCs were loaded with $5 \mu \mathrm{M}$ probe in buffers supplied by the manufacturer, and incubated in a humidified atmosphere $\left(95 \%\right.$ air, $5 \% \mathrm{CO}_{2}$ ) at $37^{\circ} \mathrm{C}$ for $30 \mathrm{~min}$. The cells were washed and measured using a luminescence spectrometer (PerkinElmer, Inc., Waltham, MA, USA) with an excitation source at $488 \mathrm{~nm}$ and an emission at $530 \mathrm{~nm}$.

Estimation of lipid peroxidation. Malondialdehyde (MDA) reacts with thiobarbituric acid to produce a fluorescent product. The level of MDA was measured in PDLSC lysates with a microplate reader at a wavelength of $535 \mathrm{~nm}$. PDLSCs at an estimated density of $2.5 \times 10^{5}$ were harvested and washed with PBS after $24 \mathrm{~h}$. The MDA was measured according to the manufacturer's protocol of the MDA detection kit (Beyotime Institute of Biotechnology, Haimen, China).
DCFDA staining. Cellular ROS production was measured using a DCFDA assay kit, as previously reported (43). Briefly, PDLSCs were incubated with $25 \mu \mathrm{M}$ DCFDA at $37^{\circ} \mathrm{C}$ for $45 \mathrm{~min}$, and then exposed to hyperosmotic medium (450 mOsM). Fluorescent images were captured using a Nikon Eclipse Ti microscope (Nikon Corporation, Tokyo, Japan). Images were captured at $\mathrm{x} 400$ magnification.

Flow cytometry. PDLSCs were stained with DCFDA as described above and resuspended in PBS. Fluorescence was measured by FACSCalibur flow cytometry (BD Biosciences). Data were analyzed and graphed using Beckman Coulter Epics XL-MCL running System II software (version 3.0; Beckman Coulter, Inc., Brea, CA, USA).

Statistical analysis. Data are presented as the mean \pm standard deviation and at least three separate experiments were performed. Student's t-tests were performed to assess significant differences between two groups, and comparisons among multiple groups were conducted using single-factor analysis of variance with Tukey's post hoc test. Statistical calculations were performed using SPSS 20.0 (IBM Corp., Armonk, NY, USA). $\mathrm{P}<0.05$ was considered to indicate a statistically significant difference.

\section{Results}

Hydrogen peroxide impairs the expression of Creb. Rat PDLSCs were isolated and characterized (Fig. S1), and were then used to examine the expression levels of Creb under oxidative stress. After treating the primary PDLSCs with $\mathrm{H}_{2} \mathrm{O}_{2}$ at concentrations of $0,62.5,125,250,500$ and $1,000 \mu \mathrm{M}$ for $24 \mathrm{~h}$, the results demonstrated that the mRNA and protein expression levels of Creb were significantly reduced upon treatment with $\mathrm{H}_{2} \mathrm{O}_{2}$ treatment in a dose-dependent manner (Fig. 1A and B), suggesting that the expression of Creb is negatively controlled by $\mathrm{H}_{2} \mathrm{O}_{2}$. Since $\mathrm{H}_{2} \mathrm{O}_{2}$ concentrations of $125 \mu \mathrm{M}$ or higher reduced Creb expression by $>50 \%, 125 \mu \mathrm{M}$ was selected as the half-maximal effective dose of $\mathrm{H}_{2} \mathrm{O}_{2}$, and this concentration was used for the following studies.

Overexpression of Creb protects PDLSCs from $\mathrm{H}_{2} \mathrm{O}_{2}$-induced cytotoxicity. To further explore the role Creb of PDLSCs, a Creb-overexpressing plasmid was constructed and transfected into isolated PDLSCs. RT-qPCR and western blotting demonstrated significant overexpression of Creb at the mRNA and protein levels (Fig. 2A and B). In the presence of $\mathrm{H}_{2} \mathrm{O}_{2}$, cell viability assessed by MTT assay in PDLSCs decreased significantly (Fig. 2C). However, PDLSCs with overexpressed Creb displayed a higher survival rate compared with wild-type PDLSCs (Fig. 2C). Meanwhile, we did not observe a significant difference in cell viability between wild-type PDLSCs and Creb over-expressing PDLSCs under normal culture conditions (Fig. 2C).

Creb overexpression attenuates $\mathrm{H}_{2} \mathrm{O}_{2}$-induced $\mathrm{ROS}$ damage. To understand how Creb rescues PDLSCs from $\mathrm{H}_{2} \mathrm{O}_{2}$-induced cytotoxicity, the ROS resulting from $\mathrm{H}_{2} \mathrm{O}_{2}$ treatment were first analyzed. Treatment with $\mathrm{H}_{2} \mathrm{O}_{2}$ caused a significant induction of ROS in PDLSCs, and overexpression of Creb significantly 

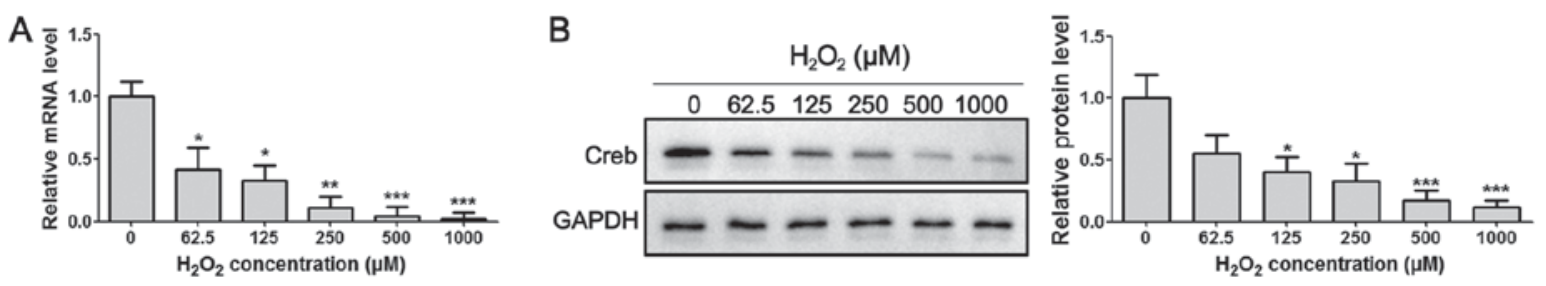

Figure 1. $\mathrm{H}_{2} \mathrm{O}_{2}$ treatment downregulates Creb expression in PDLSCs. (A) Reverse transcription-quantitative polymerase chain reaction and (B) western blot analysis of Creb expression after $24 \mathrm{~h}$ of treatment with $\mathrm{H}_{2} \mathrm{O}_{2}$ at the indicated concentrations. PDLSCs treated with an equivalent volume of PBS were used as the control. ${ }^{*} \mathrm{P}<0.05,{ }^{* *} \mathrm{P}<0.01,{ }^{* * * *} \mathrm{P}<0.001$ vs. respective control. PDLSCs, periodontal ligament stem cells; Creb, cAMP responsive element binding protein.
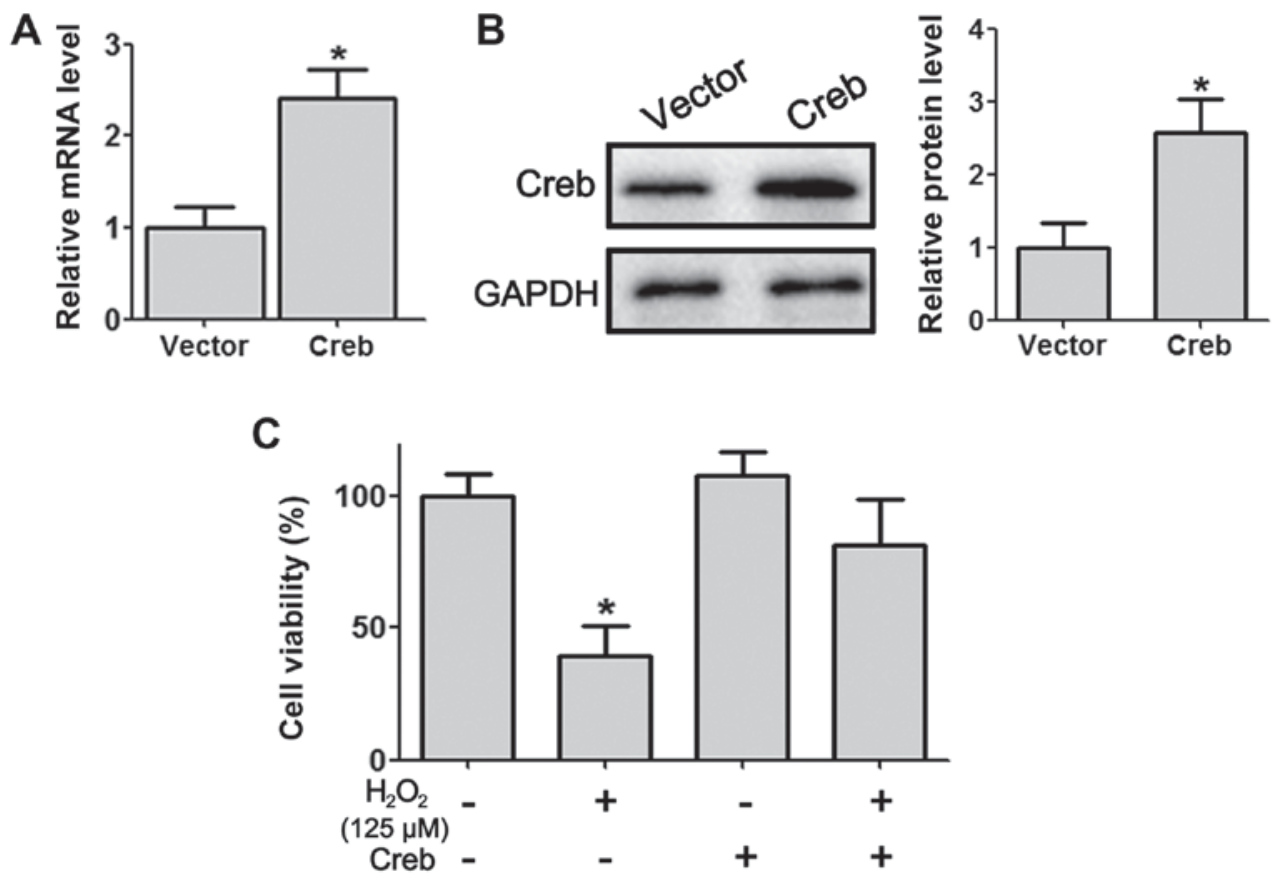

Figure 2. Overexpression of Creb protects PDLSCs from $\mathrm{H}_{2} \mathrm{O}_{2}$-induced cytotoxicity. (A) Reverse transcription-quantitative polymerase chain reaction and (B) western blot analysis illustrating Creb expression in PDLSCs transfected with pcDNA3.1 or pcDNA3.1-Creb for $48 \mathrm{~h}$. (C) A total of $48 \mathrm{~h}$ post-transfection, PDLSCs were treated with $125 \mu \mathrm{M} \mathrm{H}_{2} \mathrm{O}_{2}$ for $24 \mathrm{~h}$, and cell viability was detected by MTT assay. ${ }^{*} \mathrm{P}<0.05$ vs. respective control. PDLSCs, periodontal ligament stem cells; Creb, cAMP responsive element binding protein.

attenuated the ROS to approximately basal levels (Fig. 3A). Additionally, the MDA level induced by treatment with $\mathrm{H}_{2} \mathrm{O}_{2}$ was also recovered by the overexpression of Creb (Fig. 3B), suggesting that $\mathrm{Creb}$ protected PDLSCs from $\mathrm{H}_{2} \mathrm{O}_{2}$-induced ROS damage. Upon oxidation, $\mathrm{H}_{2}$ DCFDA becomes the highly green fluorescent DCFDA, providing an excellent tool for ROS detection. $\mathrm{H}_{2}$ DCFDA was applied to the culture media and the fluorescent signals were examined. The results demonstrated that $\mathrm{H}_{2} \mathrm{O}_{2}$ administration induced strong fluorescent signals, while Creb overexpression effectively attenuated the signals (Fig. 3C). The DCFDA fluorescence intensity was also measured by flow cytometry, and the results indicated that the cells treated with $\mathrm{H}_{2} \mathrm{O}_{2}$ exhibited high DCFDA fluorescence intensity, while the fluorescence intensity was significantly reduced in Creb-overexpressing PDLSCs (Fig. 3D).

Creb overexpression leads to activation of Creb/Bcl-2/Caspase signal. Creb is a critical regulator of the anti-apoptotic protein Bcl-2 and phosphorylated Creb protein directly induces the expression of $\mathrm{Bcl}-2$. The present results demonstrated that in
Creb-overexpressing PDLSCs, Bcl-2 expression was induced (Fig. 4). When the PDLSCs were treated with $\mathrm{H}_{2} \mathrm{O}_{2}$, the protein expression of $\mathrm{Creb}$, phosphorylated $\mathrm{Creb}$ and $\mathrm{Bcl}-2$ were all impaired while cleaved caspase-9, an initiator of apoptosis, was significantly increased (Fig. 4). In Creb-overexpressing PDLSCs, the activation of caspase-9 was efficiently attenuated due to the induction of Bcl-2 (Fig. 4), suggesting that $\mathrm{Creb} / \mathrm{Bcl}-2 /$ caspase signaling is a critical pathway that regulates apoptosis in PDLSCs.

Inhibition of ERK pathway abrogates Creb-induced ROS resistance. ERK signaling regulates Creb activity and oxidative stress modulates the phosphorylation of ERK. The results in PDLSCs demonstrated that treatment with $\mathrm{H}_{2} \mathrm{O}_{2}$ induces the expression of phosphorylated ERK, and administration of U0126, a highly selective inhibitor of ERK, resulted in the near-total ablation of phosphorylated ERK and phosphorylated Creb (Fig. 5A). In addition, Creb overexpression significantly increased the expression level of phosphorylated Creb, even under treatment with $\mathrm{H}_{2} \mathrm{O}_{2}$, while inhibition of the ERK pathway by U0126 abrogated the induced expression of 


\section{A}

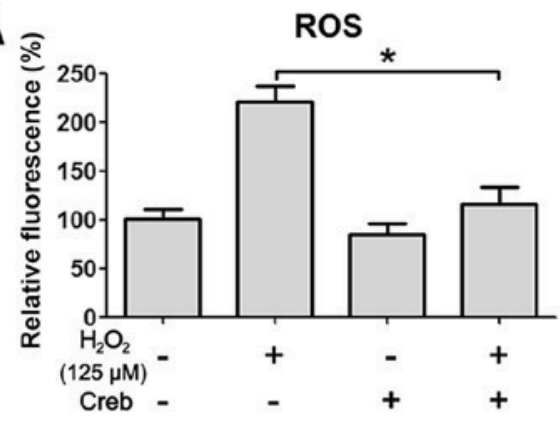

C
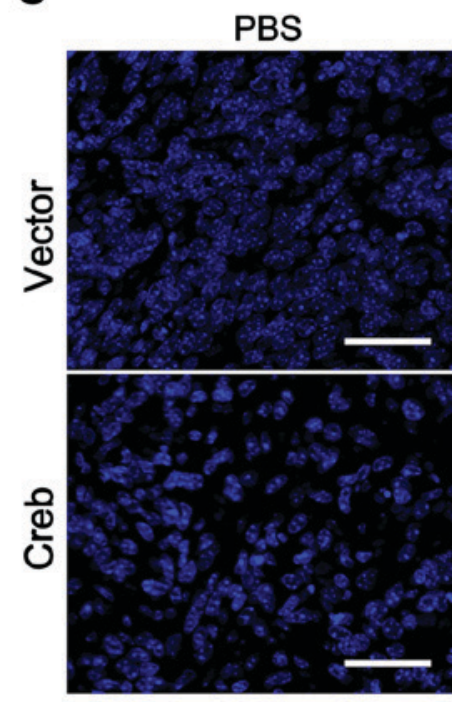

B
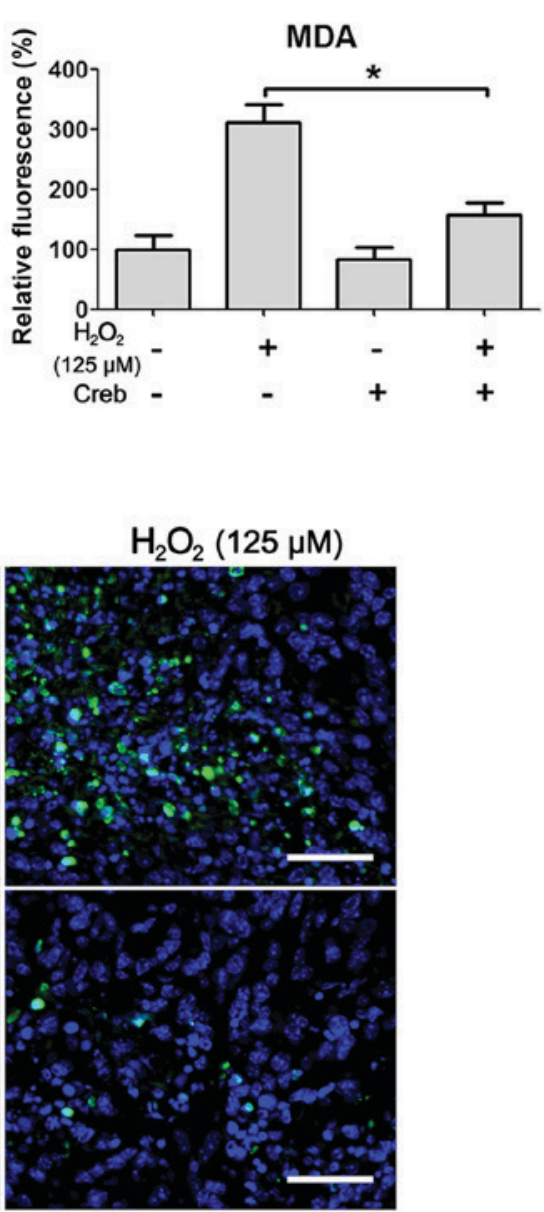

D

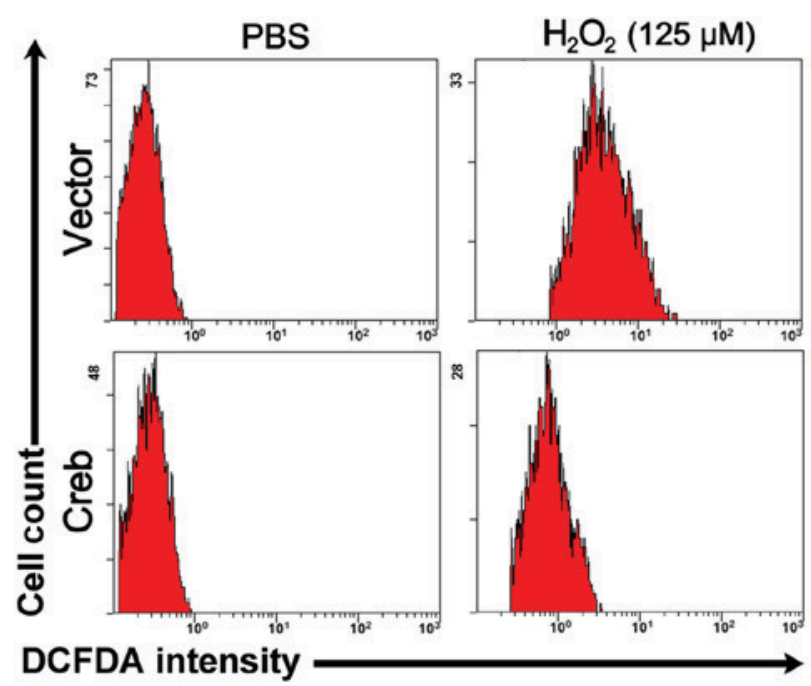

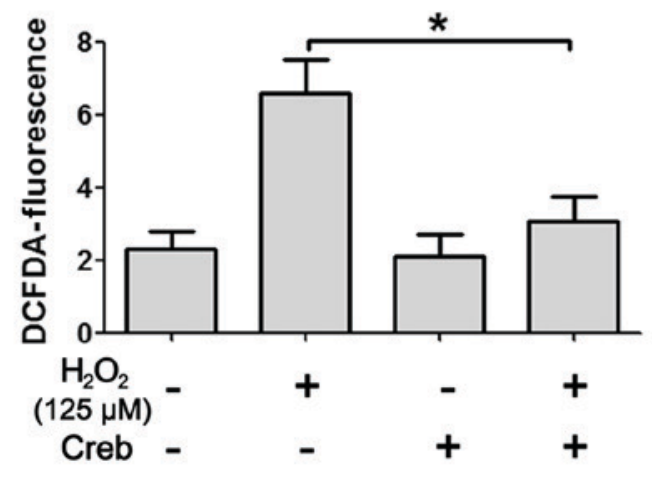

Figure 3. Creb overexpression attenuates $\mathrm{H}_{2} \mathrm{O}_{2}$-induced ROS damage. PDLSCs with/without overexpression of Creb were exposed to $\mathrm{H}_{2} \mathrm{O}_{2}$ (125 $\left.\mu \mathrm{M}\right)$ for $24 \mathrm{~h}$, and the production of (A) ROS and (B) MDA was determined. (C) DCFDA staining illustrating the production of intracellular ROS. Scale bars, $50 \mu \mathrm{m}$. (D) The fluorescence intensity of DCFDA was measured by flow cytometry. Values are presented as the mean arbitrary unit \pm standard deviation of three independent repeats. ${ }^{*}<0.05$. PDLSCs, periodontal ligament stem cells; Creb, cAMP responsive element binding protein; ROS, reactive oxygen species; MDA, malondialdehyde; DCFDA, 2',7'-dichlorodihydrofluorescein diacetate.

phosphorylated Creb (Fig. 5B). As a result, the abrogation of phosphorylated Creb resulted in a marked decrease in the cell viability of Creb-overexpressing PDLSCs (Fig. 5C), suggesting that Creb-induced ROS resistance in PDLSCs depends on ERK activity.

\section{Discussion}

Oxidative stress is accompanied by inflammation, and increasing evidence suggests that inflammation weakens the regenerative capacity of PDLSCs, although the proliferation 

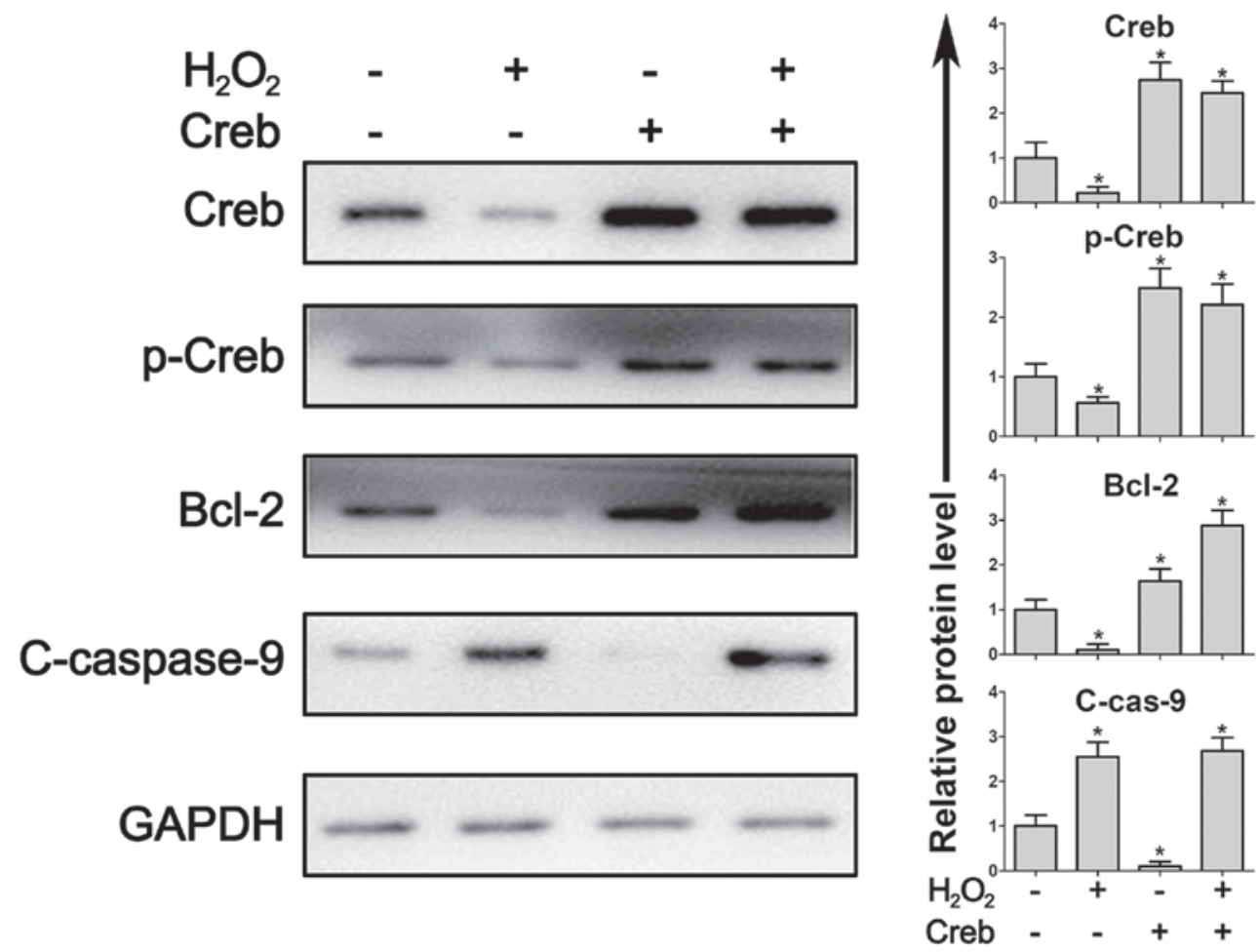

Figure 4. Overexpression of Creb leads to activation of $\mathrm{Creb} / \mathrm{Bcl}-2 / \mathrm{Caspase}$ signaling. Periodontal ligament stem cells with/without overexpression of Creb were exposed to $\mathrm{H}_{2} \mathrm{O}_{2}(125 \mu \mathrm{M})$ for $24 \mathrm{~h}$. Creb, p-Creb, Bcl-2 and c-caspase-9 were detected by western blotting. Intracellular GAPDH was used as loading control. ${ }^{*} \mathrm{P}<0.05$ vs. respective control. Creb, cAMP responsive element binding protein; $\mathrm{p}$, phosphorylated; c, cleaved; Bcl-2, apoptosis regulator Bcl-2.
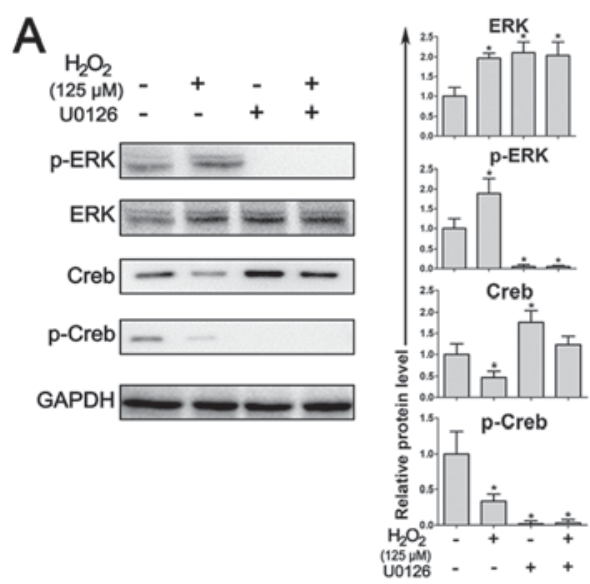

B
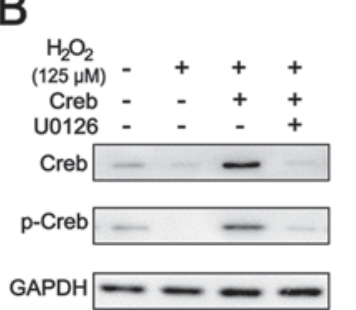

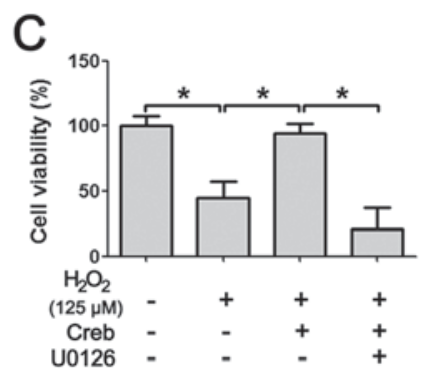

Figure 5. Inhibition of the ERK pathway abrogates Creb-induced ROS resistance. (A) Treatment with the ERK inhibitor U0126 inhibits the activation of Creb. PDLSCs with/without overexpression of Creb were exposed to $\mathrm{H}_{2} \mathrm{O}_{2}(125 \mu \mathrm{M})$ for $24 \mathrm{~h}$. ERK, p-ERK, Creb and p-Creb were detected by western blotting. (B) Inhibition of the ERK pathway attenuates Creb phosphorylation. PDLSCs with/without overexpression of Creb were treated with $\mathrm{H}_{2} \mathrm{O}_{2}(125 \mu \mathrm{M})$ for $24 \mathrm{~h}$, $10 \mu \mathrm{M}$ U0126 was used as an ERK specific inhibitor. Creb and p-Creb were detected by western blotting. Intracellular GAPDH was used as a loading control. (C) The viability of PDLSCs was detected by MTT assay. "P<0.05. ERK, extracellular signal-regulated kinase; p, phosphorylated; Creb, cAMP responsive element binding protein.

and migration of PDLSCs may be strengthened $(44,45)$. However, little is known about how PDLSCs respond to oxidative stress. As a promising cell source for regenerative medicine, it is of great importance to understand how PDLSCs are regulated under various external stimuli. In the present study, the results revealed that under treatment with $\mathrm{H}_{2} \mathrm{O}_{2}$, mRNA and protein expression of Creb were significantly reduced in a dose-dependent manner, suggesting a regulatory role of Creb in PDLSCs. More importantly, enhanced Creb expression protected PDLSCs from $\mathrm{H}_{2} \mathrm{O}_{2}$-induced cytotoxicity.
The present findings suggested that Creb expression is closely associated with the survival of PDLSCs. Modulating Creb expression may be a way of improving the in vitro culture efficiency of PDLSCs in the repair of periodontal ligaments.

A significant increase in ROS levels was observed by monitoring ROS, MDA and DCFDA intensities, suggesting that PDLSCs are sensitive to environmental stimuli. PDLSCs are very sensitive to ROS stimulation. It has been reported that the phosphatidylinositol 3-kinase/RAC- $\alpha$ serine/threonine protein kinase and $\beta$-catenin pathways are affected 
when PDLSCs are subjected to ROS stimulation. The present data revealed a de novo pathway that involves Creb protein, which shares a crosstalk with the $\beta$-catenin pathways $(42,46)$. Elevated ROS levels are associated with apoptosis and Bcl-2 is a critical regulator in ROS-associated apoptosis (47-49), suggesting that apoptotic cell death caused at least a portion of the PDLSCs cell death under the treatment with $\mathrm{H}_{2} \mathrm{O}_{2}$. Multiple results have demonstrated the role of $\mathrm{Bcl}-2$ in apoptosis and there is a need to identify the upstream regulators of Bcl-2 (50-52). The present results demonstrated that the induction of Creb expression attenuated the $\mathrm{H}_{2} \mathrm{O}_{2}$-induced ROS elevation, suggesting that Creb protects PDLSCs from apoptosis by activating $\mathrm{Bcl}-2$. As an upstream regulator of $\mathrm{Bcl}-2$, activation of $\mathrm{Creb}$ increases the expression of $\mathrm{Bcl}-2$ resulting in reduced apoptosis $(34,35)$. The present results demonstrated that Creb-mediated Bcl-2 induction was impaired by the administration of hydrogen peroxide, while apoptosis-specific c-caspase-9 was significantly increased. Upregulation of c-caspase-9 indicates an increased level of apoptosis (53). Besides, the Creb-caspase-9 axis is a critical apoptosis regulator during cancer development (54). Overexpression of Creb successfully restored the level of phosphorylated $\mathrm{Creb}$, which attenuated the expression of $\mathrm{Bcl}-2$. In the presence of Bcl-2, the expression of the apoptotic protein caspase- 9 was inhibited and the cell viability was restored. The present results suggested that inhibition of the apoptotic cascade is at least one way in which Creb is able to protect PDLSCs from oxidative stress-induced cytotoxicity.

Creb is involved in multiple signaling pathways that regulate various cellular processes (55-57). In the nervous system, Creb is a critical regulator that modulates cell death and synaptic activity through ERK phosphorylation $(58,59)$. The phosphorylation of ERK and Creb is regulated distinctly in the presence of $\mathrm{H}_{2} \mathrm{O}_{2}$ (31). Administration of $\mathrm{H}_{2} \mathrm{O}_{2}$ increases phosphorylated ERK, but decreases phosphorylated Creb (31). The present results in PDLSCs agreed with these previous findings in PC12 cells. However, phosphorylated ERK generally increases the level of phosphorylated Creb and protects neuronal cells from apoptosis $(32,33)$. According to the present results, the $\mathrm{H}_{2} \mathrm{O}_{2}$-mediated decreased in Creb and ERK-mediated Creb phosphorylation are likely to be independent from one another. However, when the ERK-specific inhibitor U0126 was applied, the expression level of phosphorylated Creb was significantly decreased, suggesting that the activation of Creb largely depends on the activation of ERK signaling and the protective role Creb in PDLSC viability is MAPK/ERK-dependent. Thus, the results of the present study revealed signaling from the upstream MAPK/ERK signals to the downstream apoptotic inhibitor Bcl-2, and that Creb serves a critical role in this signaling in PDLSCs.

In conclusion, the present results demonstrated that the transcription factor Creb is tightly regulated in PDLSCs by environmental stimuli. Meanwhile, in PDLSCs, Creb is required for the expression of the anti-apoptotic protein Bcl-2. An appropriate level of phosphorylated Creb activated by MAPK/ERK signaling protects PDLSCs from apoptotic cell death. Mechanistically, these data suggested that treatment with $\mathrm{H}_{2} \mathrm{O}_{2}$ resulted in a significant upregulation of phosphorylated ERK, which in turn caused decreases in Creb and phosphorylated Creb. Therefore, the downstream apoptotic inhibitor Bcl-2 was downregulated, resulting in PDLSC apoptosis. The present results provide insights into PDLSC maintenance and, more importantly, further illustrate that the in vitro culture of PDLSCs designed for clinical applications may be improved by modulating the expression of Creb.

\section{Acknowledgements}

Not applicable.

\section{Funding}

This study was supported by the Administration of Traditional Chinese Medicine of Zhejiang Province (grant no. 2014ZA069) and the Department of Education of Zhejiang Province (grant no. Y201534720).

\section{Availability of data and materials}

The datasets used and/or analyzed during the current study are available from the corresponding author on reasonable request.

\section{Authors' contributions}

$\mathrm{XF}, \mathrm{BS}$, and $\mathrm{YZ}$ conceived and designed the experiments. XF performed the experiments. XF and YF analyzed the data and drafted the manuscript. BS and YZ revised the manuscript. All authors read and approved the final manuscript.

\section{Ethics approval and consent to participate}

The animal care and protocols were approved by the Institutional Review Boards of Zhejiang University (Hangzhou, China; approval no. ZJUAC160809012).

\section{Patient consent for publication}

Not applicable.

\section{Competing interests}

The authors declare that they have no competing interests.

\section{References}

1. Pihlstrom BL, Michalowicz BS and Johnson NW: Periodontal diseases. Lancet 366: 1809-1820, 2005.

2. Hynes K, Menicanin D, Gronthos S and Bartold PM: Clinical utility of stem cells for periodontal regeneration. Periodontol 2000 59: 203-227, 2012.

3. Narayanan AS and Bartold PM: Biochemistry of periodontal connective tissues and their regeneration: A current perspective. Connect Tissue Res 34: 191-201, 1996.

4. Seo BM, Miura M, Gronthos S, Bartold PM, Batouli S, Brahim J, Young M, Robey PG, Wang CY and Shi S: Investigation of multipotent postnatal stem cells from human periodontal ligament. Lancet 364: 149-155, 2004.

5. Park JY, Jeon SH and Choung PH: Efficacy of periodontal stem cell transplantation in the treatment of advanced periodontitis. Cell Transplant 20: 271-285, 2011.

6. Liu Y, Zheng Y, Ding G, Fang D, Zhang C, Bartold PM, Gronthos S, Shi S and Wang S: Periodontal ligament stem cell-mediated treatment for periodontitis in miniature swine. Stem Cells 26: 1065-1073, 2008. 
7. Fu X, Jin L, Ma P, Fan Z and Wang S: Allogeneic stem cells from deciduous teeth in treatment for periodontitis in miniature swine. J Periodontol 85: 845-851, 2014.

8. Huang CY, Pelaez D, Dominguez-Bendala J, Garcia-Godoy F and Cheung HS: Plasticity of stem cells derived from adult periodontal ligament. Regen Med 4: 809-821, 2009.

9. Li Q, Ma Y, Zhu Y, Zhang T and Zhou Y: Declined expression of histone deacetylase 6 contributes to periodontal ligament stem cell aging. J Periodontol 88: e12-e23, 2017.

10. Wang Z, Feng Z, Wu G, Bai S, Dong Y and Zhao Y: In vitro studies on human periodontal ligament stem cell sheets enhanced by enamel matrix derivative. Colloids Surf B Biointerfaces 141: 102-111, 2016.

11. Kato H, Taguchi Y, Tominaga K, Kimura D, Yamawaki I, Noguchi M, Yamauchi N, Tamura I, Tanaka A and Umeda M: High glucose concentrations suppress the proliferation of human periodontal ligament stem cells and their differentiation into osteoblasts. J Periodontol 87: e44-e51, 2016.

12. He Y, Jian CX, Zhang HY, Zhou Y, Wu X, Zhang G and Tan YH: Hypoxia enhances periodontal ligament stem cell proliferation via the MAPK signaling pathway. Genet Mol Res 15, 2016. doi: $10.4238 / \mathrm{gmr15048965}$

13. E LL, Xu WH, Feng L, Liu Y, Cai DQ, Wen N and Zheng WJ: Estrogen enhances the bone regeneration potential of periodonta ligament stem cells derived from osteoporotic rats and seeded on nano-hydroxyapatite/collagen/poly(L-lactide). Int J Mol Med 37: 1475-1486, 2016

14. Chen Z and Liu HL: Restoration of miR-1305 relieves the inhibitory effect of nicotine on periodontal ligament-derived stem cell proliferation, migration, and osteogenic differentiation. J Oral Pathol Med 46: 313-320, 2017

15. Jia Q, Jiang W and Ni L: Down-regulated non-coding RNA (lncRNA-ANCR) promotes osteogenic differentiation of periodontal ligament stem cells. Arch Oral Biol 60: 234-241, 2015.

16. Saito S, Lin YC, Tsai MH, Lin CS, Murayama Y, Sato R and Yokoyama KK: Emerging roles of hypoxia-inducible factors and reactive oxygen species in cancer and pluripotent stem cells Kaohsiung J Med Sci 31: 279-286, 2015.

17. Kobayashi CI and Suda T: Regulation of reactive oxygen species in stem cells and cancer stem cells. J Cell Physiol 227: 421-430, 2012.

18. Asensi KD, Fortunato RS, dos Santos DS, Pacheco TS, de Rezende DF, Rodrigues DC, Mesquita FC, Kasai-Brunswick TH, de Carvalho AC, Carvalho DP, et al: Reprogramming to a pluripotent state modifies mesenchymal stem cell resistance to oxidative stress. J Cell Mol Med 18 824-831, 2014

19. Hachem LD, Mothe AJ and Tator CH: Glutamate increases in vitro survival and proliferation and attenuates oxidative stress-induced cell death in adult spinal cord-derived neural stem/progenitor cells via Non-NMDA ionotropic glutamate receptors. Stem Cells Dev 25: 1223-1233, 2016.

20. Denu RA and Hematti P: Effects of oxidative stress on mesenchymal stem cell biology. Oxid Med Cell Longev 2016: 2989076 , 2016.

21. Montminy MR and Bilezikjian LM: Binding of a nuclear protein to the cyclic-AMP response element of the somatostatin gene. Nature 328: 175-178, 1987.

22. Yamamoto KK, Gonzalez GA, Biggs WH III and Montminy MR Phosphorylation-induced binding and transcriptional efficacy of nuclear factor CREB. Nature 334: 494-498, 1988

23. Gonzalez GA and Montminy MR: Cyclic AMP stimulates somatostatin gene transcription by phosphorylation of CREB at serine 133. Cell 59: 675-680, 1989.

24. Gonzalez GA, Yamamoto KK, Fischer WH, Karr D, Menzel P, Biggs W III, Vale WW and Montminy MR: A cluster of phosphorylation sites on the cyclic AMP-regulated nuclear factor CREB predicted by its sequence. Nature 337: 749-752, 1989.

25. Shaywitz AJ and Greenberg ME: CREB: A stimulus-induced transcription factor activated by a diverse array of extracellular signals. Annu Rev Biochem 68: 821-861, 1999.

26. Steven A and Seliger B: Control of CREB expression in tumors: From molecular mechanisms and signal transduction pathways to therapeutic target. Oncotarget 7: 35454-35465, 2016.

27. Deak M, Clifton AD, Lucocq LM and Alessi DR: Mitogen- and stress-activated protein kinase-1 (MSK1) is directly activated by MAPK and SAPK2/p38, and may mediate activation of CREB. EMBO J 17: 4426-4441, 1998

28. Walton MR and Dragunow I: Is CREB a key to neuronal survival? Trends Neurosci 23: 48-53, 2000.
29. Dapeng L, Xiaojie L, Ping G, Yan D and Gang S: Erk1/2 signalling is involved in the differentiation of periodontal ligament stem cells to Schwann cells in dog. Arch Oral Biol 59: 487-491, 2014.

30. Wu Y, Yang Y, Yang P, Gu Y, Zhao Z, Tan L, Zhao L, Tang T and Li Y: The osteogenic differentiation of PDLSCs is mediated through MEK/ERK and p38 MAPK signalling under hypoxia. Arch Oral Biol 58: 1357-1368, 2013.

31. Zhang L and Jope RS: Oxidative stress differentially modulates phosphorylation of ERK, p38 and CREB induced by NGF or EGF in PC12 cells. Neurobiol Aging 20: 271-278, 1999.

32. Ding G, Zhao J and Jiang D: Allicin inhibits oxidative stress-induced mitochondrial dysfunction and apoptosis by promoting PI3K/AKT and CREB/ERK signaling in osteoblast cells. Exp Ther Med 11: 2553-2560, 2016.

33. Park EM and Cho S: Enhanced ERK dependent CREB activation reduces apoptosis in staurosporine-treated human neuroblastoma SK-N-BE(2)C cells. Neurosci Lett 402: 190-194, 2006.

34. Wilson BE, Mochon E and Boxer LM: Induction of bcl-2 expression by phosphorylated CREB proteins during B-cell activation and rescue from apoptosis. Mol Cell Biol 16: 5546-5556, 1996.

35. Wang P, Yan H and Li JC: CREB-mediated Bcl-2 expression in trichosanthin-induced Hela cell apoptosis. Biochem Biophys Res Commun 363: 101-105, 2007.

36. Granville DJ and Gottlieb RA: Mitochondria: Regulators of cell death and survival. ScientificWorldJournal 2: 1569-1578, 2002.

37. Karabulut AB, Karadag N, Gurocak S, Kiran T, Tuzcu M and Sahin K: Apricot attenuates oxidative stress and modulates of Bax, Bcl-2, caspases, NFk-B, AP-1, CREB expression of rats bearing DMBA-induced liver damage and treated with a combination of radiotherapy. Food Chem Toxicol 70: 128-133, 2014.

38. Hui K, Yang Y, Shi K, Luo H, Duan J, An J, Wu P, Ci Y, Shi L and $\mathrm{Xu}$ C: The p38 MAPK-regulated PKD1/CREB/Bcl-2 pathway contributes to selenite-induced colorectal cancer cell apoptosis in vitro and in vivo. Cancer Lett 354: 189-199, 2014.

39. Favata MF, Horiuchi KY, Manos EJ, Daulerio AJ, Stradley DA, Feeser WS, Van Dyk DE, Pitts WJ, Earl RA, Hobbs F, et al: Identification of a novel inhibitor of mitogen-activated protein kinase kinase. J Biol Chem 273: 18623-18632, 1998.

40. DeSilva DR, Jones EA, Favata MF, Jaffee BD, Magolda RL, Trzaskos JM and Scherle PA: Inhibition of mitogen-activated protein kinase kinase blocks $\mathrm{T}$ cell proliferation but does not induce or prevent anergy. J Immunol 160: 4175-4181, 1998.

41. Gay IC, Chen S and MacDougall M: Isolation and characterization of multipotent human periodontal ligament stem cells. Orthod Craniofac Res 10: 149-160, 2007.

42. Livak KJ and Schmittgen TD: Analysis of relative gene expression data using real-time quantitative PCR and the 2(-Delta Delta C(T)) method. Methods 25: 402-408, 2001

43. Deng R, Hua X, Li J, Chi W, Zhang Z, Lu F, Zhang L, Pflugfelder SC and Li DQ: Oxidative stress markers induced by hyperosmolarity in primary human corneal epithelial cells. PLoS One 10: e0126561, 2015.

44. Liu N, Shi S, Deng M, Tang L, Zhang G, Liu N, Ding B, Liu W, Liu Y, Shi H, et al: High levels of $\beta$-catenin signaling reduce osteogenic differentiation of stem cells in inflammatory microenvironments through inhibition of the noncanonical Wnt pathway. J Bone Miner Res 26: 2082-2095, 2011.

45. Park JC, Kim JM, Jung IH, Kim JC, Choi SH, Cho KS and Kim CS: Isolation and characterization of human periodontal ligament (PDL) stem cells (PDLSCs) from the inflamed PDL tissue: In vitro and in vivo evaluations. J Clin Periodontol 38: 721-731, 2011.

46. Mittag S, Valenta T, Weiske J, Bloch L, Klingel S, Gradl D, Wetzel F, Chen Y, Petersen I, Basler K and Huber O: A novel role for the tumour suppressor Nitrilase1 modulating the Wnt/ß-catenin signalling pathway. Cell Discov 2: 15039, 2016.

47. Greenlund LJ, Deckwerth TL and Johnson EM Jr: Superoxide dismutase delays neuronal apoptosis: A role for reactive oxygen species in programmed neuronal death. Neuron 14: 303-315, 1995.

48. Jacobson MD and Raff MC: Programmed cell death and Bcl-2 protection in very low oxygen. Nature 374: 814-816, 1995.

49. Buttke T and Sandstrom PA: Redox regulation of programmed cell death in lymphocytes. Free Radic Res 22: 389-397, 1995.

50. Yue X, Zhao Y, Huang G, Li J, Zhu J, Feng Z and Hu W: A novel mutant p53 binding partner BAG5 stabilizes mutant p53 and promotes mutant p53 GOFs in tumorigenesis. Cell Discov 2: $16039,2016$. 
51. Ahmed KA and Xiang J: mTORC1 regulates mannose-6-phosphate receptor transport and T-cell vulnerability to regulatory $\mathrm{T}$ cells by controlling kinesin KIF13A. Cell Discov 3: 17011, 2017.

52. Narayanan N, Wang Z, Li L and Yang Y: Arginine methylation of USP9X promotes its interaction with TDRD3 and its anti-apoptotic activities in breast cancer cells. Cell Discov 3: 16048, 2017.

53. Li P, Nijhawan D, Budihardjo I, Srinivasula SM, Ahmad M, Alnemri ES and Wang X: Cytochrome $\mathrm{c}$ and dATP-dependent formation of Apaf-1/caspase-9 complex initiates an apoptotic protease cascade. Cell 91: 479-489, 1997.

54. Das TP, Suman S, Alatassi H, Ankem MK and Damodaran C: Inhibition of AKT promotes FOXO3a-dependent apoptosis in prostate cancer. Cell Death Dis 7: e2111, 2016.

55. Shi P, Liu W, Tala, Wang H, Li F, Zhang H, Wu Y, Kong Y, Zhou Z, Wang C, et al: Metformin suppresses triple-negative breast cancer stem cells by targeting KLF5 for degradation. Cell Discov 3: 17010, 2017.

56. Wu R, Liu XM, Sun JG, Chen H, Ma J, Dong M, Peng S, Wang JQ, Ding JQ, Li DH, et al: DJ-1 maintains energy and glucose homeostasis by regulating the function of brown adipose tissue. Cell Discov 3: 16054, 2017.
57. Lv S, Qiu X, Li J, Li W, Zhang C, Zhang ZN and Luan B: Suppression of CRTC2-mediated hepatic gluconeogenesis by TRAF6 contributes to hypoglycemia in septic shock. Cell Discov 2: 16046, 2016.

58. Cao N, Liao T, Liu J, Fan Z, Zeng Q, Zhou J, Pei H, Xi J, He L, Chen L, et al: Clinical-grade human umbilical cord-derived mesenchymal stem cells reverse cognitive aging via improving synaptic plasticity and endogenous neurogenesis. Cell Death Dis 8: e2996, 2017.

59. Sun XY, Tuo QZ, Liuyang ZY, Xie AJ, Feng XL, Yan X, Qiu M, Li S, Wang XL, Cao FY, et al: Extrasynaptic NMDA receptor-induced tau overexpression mediates neuronal death through suppressing survival signaling ERK phosphorylation. Cell Death Dis 7: e2449, 2016.

This work is licensed under a Creative Commons Attribution-NonCommercial-NoDerivatives 4.0 International (CC BY-NC-ND 4.0) License. 